\title{
A Bimodal Search Strategy for SETI
}

\author{
S. Gulkis and E. T. Olsen \\ Planetary Atmospheres Section \\ J. Tarter \\ University of California, Berkeley
}

\begin{abstract}
This report describes the search strategy and resultant observational plan which have been developed to carry out a comprehensive Search for Extraterrestrial Intelligence (SETI) over that portion of the electromagnetic spectrum known as the terrestrial microwave window. The limiting sensitivity achieved has been parameterized and calculated for DSN antennas as well as several radio astronomy observatories. A brief description of the instrumentation to be employed in the search and the classes of signals to be looked for is given.
\end{abstract}

\section{Introduction}

Since Coconni and Morrison (Ref. 1) first published their classic paper on the use and detectability of the $21-\mathrm{cm}$ wavelength for SETI, there have been many theoretical investigations of the likelihood of intelligent life appearing in our galaxy and a few restricted search programs carried out. But regardless of the number of communicative civilizations in our galaxy, we still do not know where to point our antennas, what frequency to listen on, or what type of signal to search for. To determine these, we must engage in an extensive and systematic search program.

About four years ago, groups at the NASA Ames Research Center (ARC) in Mountain View, Califomia, and at the Jet Propulsion Laboratory (JPL) in Pasadena, California set out jointly to develop a search strategy which had as its objective to greatly expand the parameter space which has been observed to date, and to accomplish this with existing telescopes. The search strategy developed by these groups is based on observations to be carried out primarily in the terrestrial microwave window (defined here to be the spectral range from $600 \mathrm{MHz}$ to $25 \mathrm{GHz}$ ). This is the spectral region in which the sky brightness is minimum, which contains the "water hole" (Oliver, Ref. 2) and microwave water line frequencies, and for which instrumentation is readily available.

The search strategy assumes that signals of extraterrestrial origin may be narrowband transmissions which are continuously present or pulsed. The proposed strategy will be most successful at detecting those types of signals which are designed to be easily detectable but will nevertheless retain some sensitivity to more complex types of signals. The observational program embodied in the ARC/JPL plan is bimodal in character so as to cover a wide range of possibilities (Seeger, Ref. 3). One goal of the program is to survey the entire sky over a wide range of frequency to a relatively constant flux level (Murray et al., Ref. 4). This survey ensures that all potential life sites are observed to some limiting 
equivalent isotropic radiated power (EIRP) depending upon their distance. A second goal is to survey a set of potential transmission sites selected $a$ priori to be especially promising, achieving very high sensitivity over a smaller range of frequency (Oliver and Billingham, Ref. 5). The purpose of this paper will be to discuss the various aspects of these two complementary observational goals embodied in the ARC/JPL search strategy. Table 1 summarizes the principal observing parameters of these two approaches.

\section{Sensitivity Limits for Existing Telescopes}

It is presumed at the onset of the ARC/JPL program that existing radio telescopes, equipped with state-of-the-art receivers and data processing devices, will have both the sensitivity to explore the vicinity of nearby stars for transmitters similar to earth's, and to explore the entire galaxy for more powerful signals or for signals beamed at us. In this section, we examine this assumption on a quantitative basis.

An isotropic transmitter with power $P_{T}$ at a distance $r$ will create a flux

$$
\phi=P_{T} / 4 \pi r^{2} .
$$

The power received by an antenus of physical area $A$ and aperture efficiency $\eta$, from a source which produces a flux $\phi$, is given by

$$
P=\phi \eta A \text {. }
$$

This power can be detected when it exceeds the inherent noise fluctuations of the system. For a postintegration power detection receiver with equivalent input noise temperature $T$ and IF bandwidth $b$, the standard deviation of the noise fluctuations is given by

$$
\sigma=k T \sqrt{\frac{b}{t}} .
$$

where $k$ is Boltzman's constant and $t$ is the integration time. Thus, detection of a signal is possible when the signal power $P$ exceeds the noise power $\sigma$ by some specified factor $\alpha$, determined by the allowable probability of a false alarm. Hence, we have the condition for detectability given by

$$
P \geqslant \alpha \sigma=\alpha k T \sqrt{\frac{b}{t}} .
$$

Combining Eqs. (2) and (4), we can write for the minimum detectable flux (in $\mathrm{W} / \mathrm{m}^{2}$ )

$$
\phi=\frac{\alpha k T}{\eta A} \sqrt{\frac{b}{t}}
$$

Table 2 gives sensitivity limits for some existing telescopes instrumented with low noise receivers. The parameters used are as follows:

$$
\begin{aligned}
\alpha & =5 \\
b & =1 \mathrm{~Hz} \\
\tau & =1 \mathrm{~s} \text { and } 1000 \mathrm{~s} \\
\eta & =0.5
\end{aligned}
$$

If 1000 seconds is the integration time on each star, sensitivities in the range $4.9 \times 10^{-27} \mathrm{~W} / \mathrm{m}^{2}$ to $3.3 \times 10^{-25}$ $\mathrm{W} / \mathrm{m}^{2}$ are achievable from the largest to smallest telescope for spectral resolution of $1 \mathrm{~Hz}$. The combined frequency resolution and sensitivity $(1 / 2$ jansky in a $1-\mathrm{Hz}$ bandwidth for Arecibo) fall far outside that which has been used in radio astronomy.

From Eq. (1) the flux received at the earth from a transmitter $r$ light-years away, per watt of equivalent isotropic radiated power, is

$$
\phi=\frac{8.89 \times 10^{-34} E I R P}{r^{2}} \mathrm{~W} / \mathrm{m}^{2} .
$$

We may write for the range of a detectable signal

$$
r=\left[\frac{8.89 \times 10^{-34}(E I R P)(\eta)(A)}{\alpha k T} \sqrt{\frac{t}{b}}\right]^{1 / 2}
$$

Figure 1 shows the minimum detectable EIRP as a function of distance for a typical sky survey $(\alpha=5, b=32 \mathrm{~Hz}, \tau=1 \mathrm{~s})$ carried out with a 34-m telescope and for two telescopes used in a discrete source survey $(\alpha=5, b=1 \mathrm{~Hz}, \tau=1000 \mathrm{sec})$. We see from this figure that the Arecibo telescope can detect $10^{8} \mathrm{~W}$ (EIRP) out to about 4 light-years, the distance to the nearest stars. Transmitters at the level of the strongest TV stations, $10^{5}-10^{7} \mathrm{~W}$ (EIRP), can be detected only if they are situated less than 2 light-years away. A transmitter equivalent to the most powerful radar systems used on earth, $10^{13} \mathrm{~W}$ (EIRP), can be detected by the sky survey at a distance of 22 light-years and by the discrete source survey at a distance of 1348 light-years. 


\section{Bimodal Observational Plan}

\section{A. Discrete Source Mode}

The a priori site survey is designed to observe 773 stars which have been identified within 25 parsecs of the sun to be of spectral type $F, G$, or $K$ and luminosity class $V$. The frequency range to be covered will be 1.2 to $3 \mathrm{GHz}$ and as many spot bands between 3 and $25 \mathrm{GHz}$ as time permits. This spectral region includes the water hole, 1.4 to $1.7 \mathrm{GHz}$, which has been suggested as a preferred frequency band for an interstellar search (Oliver, Ref. 2).

The sensitivity level which can be achieved depends on both the telescope used and the integration time. Table 2 gives typical sensitivities that can be achieved with the Arecibo antenna and with several smaller DSN antennas. Only 244 stars are visible to the Arecibo telescope; the other 529 stars must be observed using telescopes more than a factor of 3 smaller in diameter. The integration time required to achieve a constant sensitivity varies as the inverse fourth power of the telescope diameter. Thus, no attempt will be made to achieve a constant sensitivity search, as this would require $\sim 260$ times as much observing time on a 64-m antenna as on the Arecibo antenna. Rather, all stars shall be observed for approximately the same integration duration.

\section{B. Sky Survey}

The sky survey will search the entire celestial sphere over the frequency range $1.2<f<10 \mathrm{GHz}$ inclusive and as many spot bands between 10 and $25 \mathrm{GHz}$ as time permits. In order to better understand the constraints on time and sensitivity of a sky survey, it is convenient to modify Eq. (5) by substituting for the integration time $t$ the amount of time required to sweep the telescope primary beam past a location on the celestial sphere. Let $\omega$ be the angular tracking rate in deg/s. Then the time required to move a half-power beamwidth is

$$
\xi=70 c / f D \omega
$$

in seconds, where $D$ is the diameter of the telescope, $f$ is the observation frequency, and $c$ is the velocity of light. Since $A=$ $\pi D^{2} / 4$ for a circular aperture, Eq. (5) becomes:

$$
\phi=\left(4 \alpha k T_{s} / \pi \eta\right) \sqrt{\omega b f / 70 c D^{3}}
$$

in $\mathrm{W} / \mathrm{m}^{2}$.

Now the time required to survey a fraction $g$ of the sky is

$$
T_{i}=4 \pi g \xi / \Omega_{i}
$$

where the solid angle of the beam is

$$
\Omega_{i}=\epsilon c^{2} / \eta A f_{i}^{2}
$$

and $\epsilon$ is the beam efficiency. Substituting Eq. (11) into (10) and assuming a circular aperture, the time to survey at a particular frequency setting is found:

$$
T_{i}=\left(70 \mathrm{~g} \pi^{2} \eta D f_{i} /(c \omega \epsilon) .\right.
$$

The time to carry out a complete survey between the frequency limits $f_{L}$ and $f_{u}$ depends on both the bandpass $B$ of the multichannel spectrum analyzer and the scan rate. The bandpass $B$ is equal to the product of the number of channels in the spectrum analyzer and the single channel bandwidth $(b)$. An attractive operational procedure is to operate at a constant angular tracking rate. This choice results in a sensitivity which varies as the square root of the frequency. This leads to the following expression for the time to carry out a survey between the frequency limits $f_{L}$ and $f_{u}$

$$
T=\left[\left(70 g \pi^{2} \eta D\right) /(2 c \omega B \epsilon)\right]\left(f_{u}^{2}-f_{L}^{2}\right) .
$$

Assuming that the survey is carried out using a $34-\mathrm{m}$ telescope which scans at a rate of $0.2 \%$, the time required to survey the entire sky at a single frequency setting is

$$
T_{i}=3 \sqrt{f_{G H z}} \text { days }
$$

if the scans are separated by one half-power beam width. The instantaneous bandpass of a $2^{23}$ channel analyzer operating at a spectral resolution of $32 \mathrm{~Hz}$ is $268.4 \mathrm{MHz}$. Thus the time required to survey the entire celestial sphere over the frequency range $1.2 \leqslant f \leqslant 10 \mathrm{GHz}$ is

$$
T \approx 1.6 \text { years }
$$

The sensitivity achieved in such a survey will be

$$
\phi=2.0 \times 10^{-24} \alpha \sqrt{f_{G H z}} \mathrm{~W} / \mathrm{m}^{2} .
$$

\section{Instrumentation}

Figure 2 shows a block diagram of a system instrumented for SETI observations. The complete system consists of the radio telescope; orthogonally polarized feeds, low noise receivers, a large multichannel spectrum analyzer (MCSA) and associated accumulators, processor, and various recording devices. 
The crucial new instrumentation which enables this search to greatly expand on previous searches is the large multichannel spectral analyzer. Such devices will make it possible to examine large numbers of frequencies simultaneously. A digital spectrum analyzer with $300 \mathrm{MHz}$ of bandwidth and a million channels is currently under construction at the Jet Propulsion Laboratory (Morris and Wilck, Ref. 6). This device produces FFT transforms in $10-\mathrm{MHz}$ sections with $300-\mathrm{Hz}$ resolution. One such device designed at Stanford University in collaboration with the Ames Research Center and JPL yields $10^{7}$ channels over $8.4 \mathrm{MHz}$ with a resolution of $1 \mathrm{~Hz}$ or $10^{7}$ channels over $268 \mathrm{MHz}$ with a resolution of $32 \mathrm{~Hz}$.

Figures 3 and 4 show schematic block diagrams of the sky survey and discrete source survey devices which consist of two stages of digital bandpass filters followed by digital FFT (Fast Fourier Transform) processors. The primary spectral resolutions originating in this design are $8.4 \mathrm{MHz}, 65.5 \mathrm{kHz}, 1 \mathrm{kHz}$, $32 \mathrm{~Hz}$ and $1 \mathrm{~Hz}$. Synthetic spectral resolutions may be generated by combining samples in frequency and in time, and the MCSA will be designed to output power spectra over the range of spectral resolution from $1 / 4 \mathrm{~Hz}$ to $4 \mathrm{kHz}$ in steps which are multiples of 2 . This resolution tree allows a more nearly optimum match to a wide range of continuous and pulsed signal bandwidths. The bandpass filter design ensures spectral isolation to the $1-\mathrm{kHz}$ level $(256 \mathrm{~Hz}$ counting synthetic frequencies); i.e., a strong interfering signal will not "splatter" more than this amount beyond its own bandwidth. The use of FFT processors at the high-resolution end of the chain minimizes memory required in the hardware. The MCSA architecture is highly multiplexed so that the actual board level parts count is minimal.

The MCSA is designed to be microprogrammable, which allows the characteristics of the bandpass filters and the hardware thresholding levels $(\alpha)$ to be changed through software commands; in addition the nature of the transform performed in the fast processors may be altered by software commands. The test as to whether the power appearing in a bin exceeds a preset threshold will be carried out in the MCSA at high speed. The data corresponding to threshold crossings will be passed over to the decision processor for further tests.

The MCSA and decision processor will be sensitive to three distinct classes of signals: (1) a continuously present, nondrifting signal of the order of the mean noise level, (2) a periodically pulsed signal of the order of 10 times the mean noise level and which may be drifting, and (3) a continuously present, drifting signal of the order of the mean noise level.

Class (1) signals are the easiest to detect, requiring only a threshold test on the accumulated spectrum at the end of an integration period. Class (2) signals are more demanding on the hardware/software. Their detection requires a threshold test for each sample. Data exceeding thresholds is stored over a period of observation and then the decision processor must examine the resulting matrix for periodicity and drift. The sensitivity achieved will depend upon the number of false alarms which the system can handle. Class (3) signals require large, high-speed memory modules, for each sample spectrum must be stored over some chosen period. Sensitivity is enhanced through removal of frequency drift before accumulation and thresholding. Of course, the processor must perform this manipulation over a range of drifts.

\section{Summary Remarks}

It is presently believed that a number of large spectrum analyzers could be built and used at existing radio observatories to carry out a significant SETI program. While this program is still in its conceptual stages, we believe that a field test program could begin as early as $1980-1981$ using a smaller version of the large spectrum analyzers and other more traditional hardware. The immediate goals are to learn more about the radio frequency background (interference) at high spectral resolution and to develop signal detection algorithms which efficiently recognize the presence of a signal and automatically attempt to identify and classify it. It is expected that full-scale instrumentation development could begin in 1982 and an operational system placed in the field in 1984. 


\section{Acknowledgment}

We thank our colleagues Dr. B. Oliver and Dr. A. Peterson and members of the SETI groups at the Ames Research Center and the Jet Propulsion Laboratory for their many contributions to the ideas reflected in this paper.

\section{References}

1. Cocconi, G., and Morrision, P., "Searching for Interstellar Communications," Nature, 184 , pp. 844 (1959).

2. Oliver, B. M., "Rationale for the Water Hole," Acta Astronautica, 6, No. 1-2, pp. 71.79 (1979).

3. Seeger, C. L., "Strategic Considerations in SETI, and a Microwave Approach," Acta Astronautica, 6, No. 1-2, pp. 105-127 (1979).

4. Murray, B., Gulkis, S., and Edelson, R. E., "Extraterrestrial Intelligence: An Observational Approach," Science, 199, pp. 485-492 (1978).

5. Oliver, B. M., and Billingham, J., Project Cyclops, NASA CR114445 (1972).

6. Morris, G. A., and Wilck, H. C., "JPL $2^{20}$ Channel $300 \mathrm{MHz}$ Bandwidth Digital Spectrum Analyzer," DSN Progress Report 42-46, Jet .Propulsion Laboratory, Pasadena, Calif., pp. 57-61 (1978). 
Table 1. Search strategy observing parameters

\begin{tabular}{|c|c|c|}
\hline Parameter & All-sky survey & Selected site survey \\
\hline Coverage & $4 \pi$ steradians & $\sim 800$ beam areas \\
\hline Sensitivity limit & $\sim 10^{-23} \sqrt{f_{G H z}} \mathrm{~W} / \mathrm{m}^{2}$ & $10^{-25}-10^{-27} \mathrm{~W} / \mathrm{m}^{2}$ \\
\hline Frequency range & $\begin{array}{l}1-10 \mathrm{GHz} \\
+ \text { spot bands }\end{array}$ & $\begin{array}{l}1-3 \mathrm{GHz} \\
+ \text { spot bands }\end{array}$ \\
\hline Spectral resolution & $32 \mathrm{~Hz}$ & $1 \mathrm{~Hz}$ \\
\hline $\begin{array}{l}\text { Integration } \\
\text { time/beam area }\end{array}$ & $0.3-3 \mathrm{~s}$ & $100-1000 s$ \\
\hline $\begin{array}{l}\text { Total integration } \\
\text { time for survey }\end{array}$ & $1.6 \mathrm{yI}$ & $\sim 0.5 \mathrm{yr}$ \\
\hline Aperture & $34-m$ & $>64-\mathrm{m}$ \\
\hline
\end{tabular}

Table 2. Typical tolescope sensitivities

\begin{tabular}{|c|c|c|c|c|}
\hline \multirow[b]{2}{*}{ Telescope } & \multirow[b]{2}{*}{$T_{s}, \mathrm{~K}$} & \multirow{2}{*}{$A, \mathrm{~m}^{2}$} & \multicolumn{2}{|c|}{$\phi\left(\mathrm{W} / \mathrm{m}^{2}\right)$} \\
\hline & & & $\tau=1 \mathrm{~s}$ & $\tau=1000 \mathrm{~s}$ \\
\hline Arecibo & 40 & 35633 & $1.55 \times 10^{-25}$ & $4.89 \times 10^{-27}$ \\
\hline $\begin{array}{l}\text { Greenbank } \\
93-\mathrm{m}\end{array}$ & 30 & 6808 & $6.07 \times 10^{-25}$ & $1.92 \times 10^{-26}$ \\
\hline $\begin{array}{l}\text { Parkes or } \\
\text { DSN 64-m }\end{array}$ & 20 & 3217 & $8.58 \times 10^{-25}$ & $2.71 \times 10^{-26}$ \\
\hline Ohio State & 40 & 2221 & $2.48 \times 10^{-24}$ & $7.86 \times 10^{-26}$ \\
\hline $\begin{array}{l}\text { Stanford } \\
\text { 5-element } \\
\text { interferometer }\end{array}$ & 20 & 1313 & $2.10 \times 10^{-24}$ & $6.65 \times 10^{-26}$ \\
\hline DSN 34-m & 20 & 908 & $3.04 \times 10^{-24}$ & $9.61 \times 10^{-26}$ \\
\hline $\begin{array}{l}\text { Greenbank } \\
\text { or DSN 26-m }\end{array}$ & 20 & 527 & $5.24 \times 10^{-24}$ & $1.66 \times 10^{-25}$ \\
\hline $\begin{array}{l}\text { Stanford } \\
18-\mathrm{m}\end{array}$ & 20 & 263 & $1.05 \times 10^{-23}$ & $3.32 \times 10^{-25}$ \\
\hline
\end{tabular}




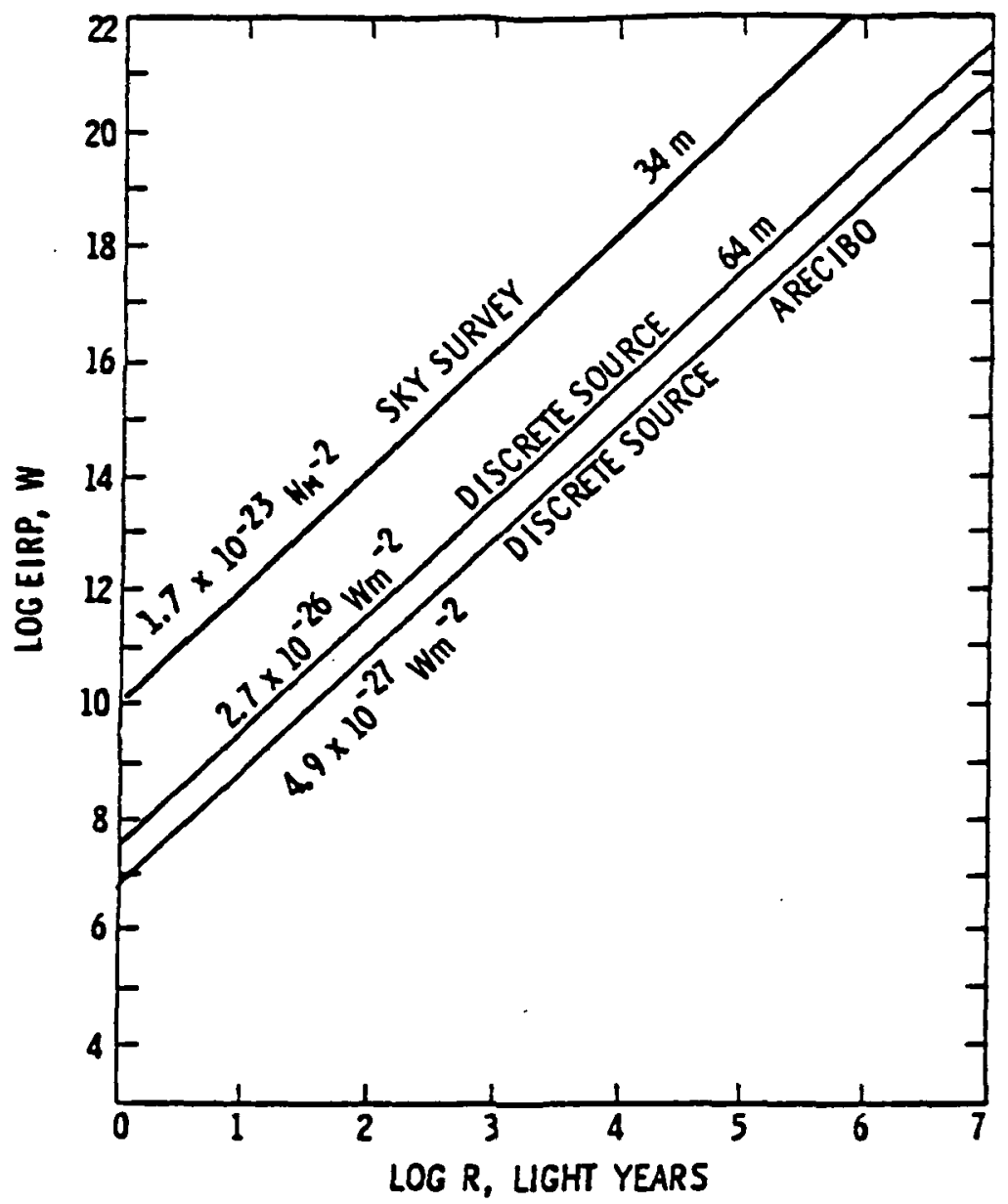

Fig. 1. The minimum equivalent isotropic radiated power detectable $a 8$ a function of distance from the transmitter for a signal-to-nolse ratio equal to five. The all-sky survey is assumed to be carried out at a spectral resolution of $32 \mathrm{~Hz}$ and a 1-8 integration perlod on a 34-m telescope. The discrete source survey is assumed to be carried out at a spectral resolution of $1 \mathrm{~Hz}$ and a 1000-s integration period on a $64 \mathrm{~m}$ telescope and the Arecibo telescope 


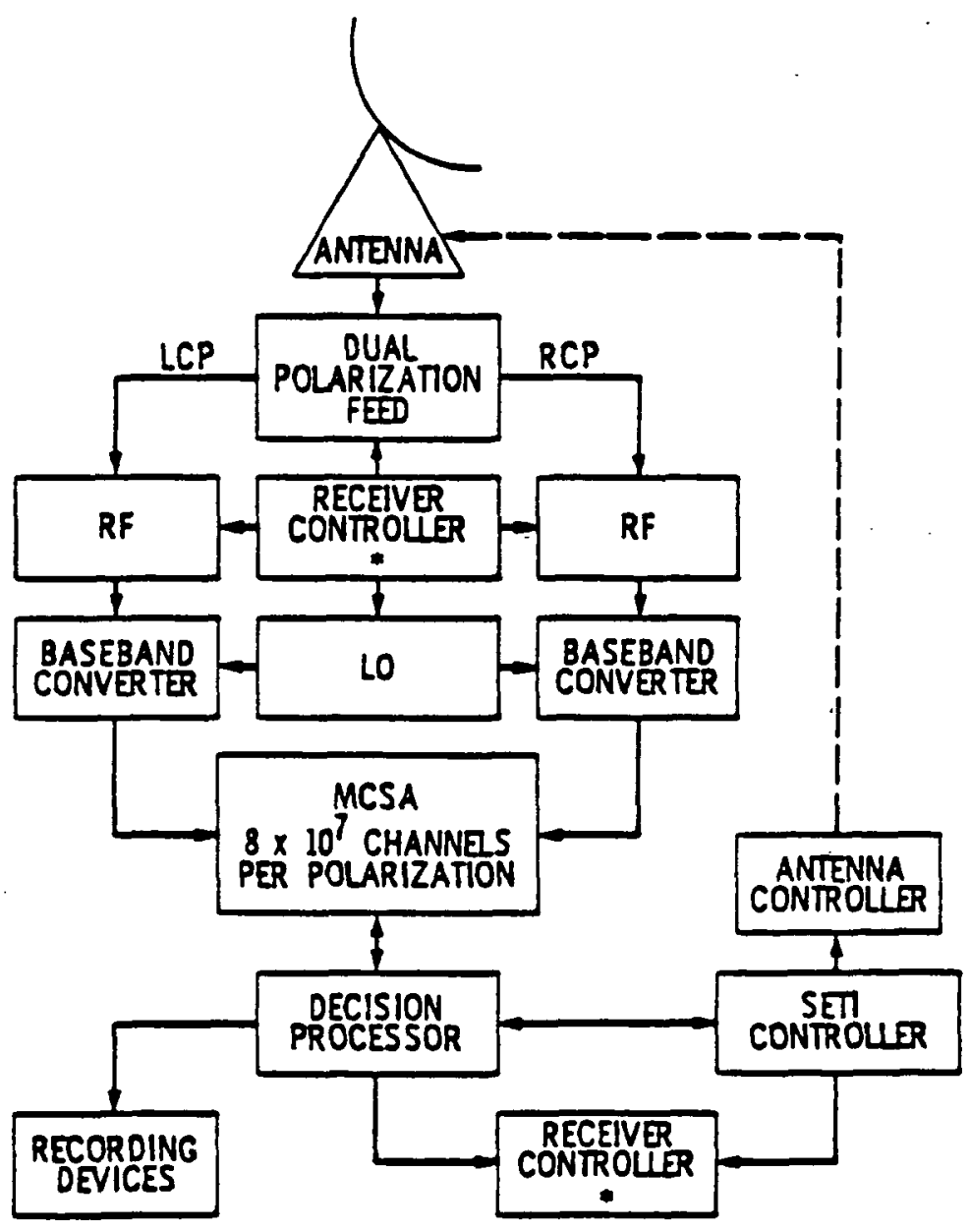

Fig. 2. A block diagram of a system instrumented to carry out a search for extraterrestrial intelligence. The system must be capable of processing data on-line to discard data of no archival value 


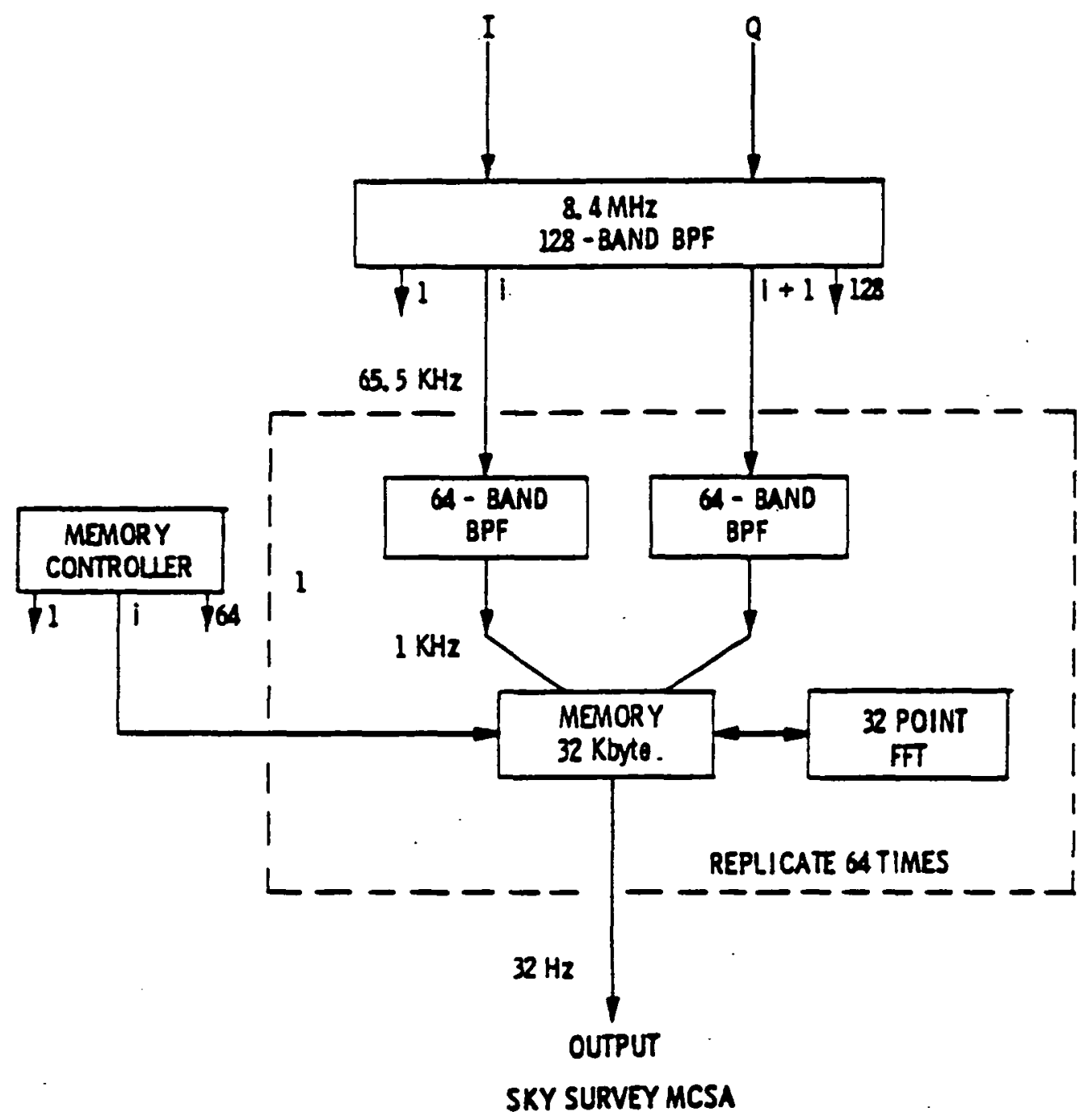

Fig. 3. A schematic block dlagram of the multichannel spectrum analyzer designed for the allshy survey. It is designed to cover a $268.4-\mathrm{MHz}$ instantaneous bandpass at 32- $\mathrm{Hz}$ resolution 


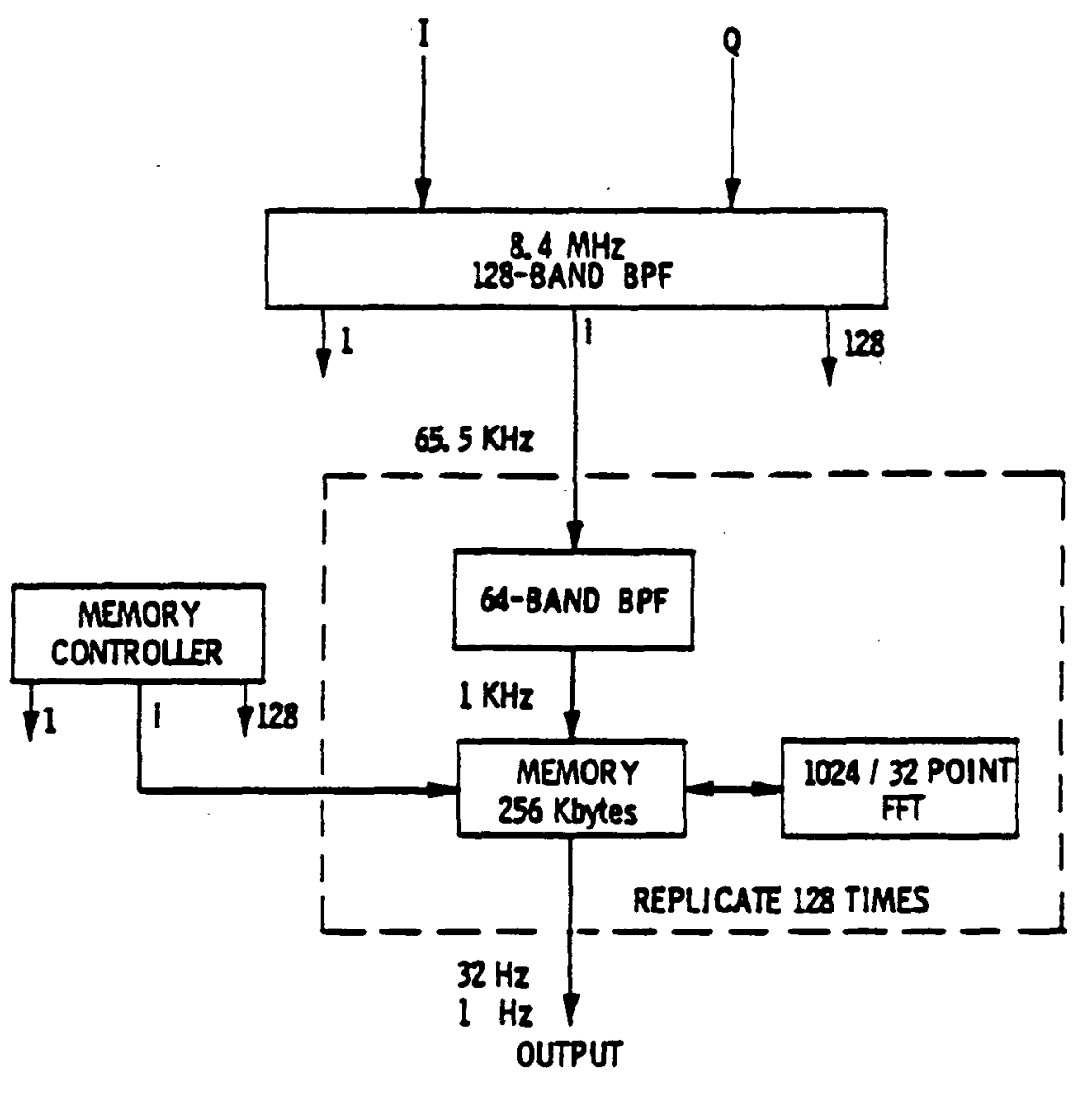

\section{DISCRETE SOURCE MCSA}

Fig. 4. A schematic block diagram of the multichannel spectrum analyzer designed for the discrete source survey. It is designed to cover a 8.4-MHz instantaneous bandpass at 1- $\mathrm{Hz}$ resolution 